\title{
Phase diagram of the integer quantum Hall effect
}

\author{
D. N. Sheng and Z. Y. Weng \\ Texas Center for Superconductivity, University of Houston, Houston, Texas 77204-5506
}

(Received 31 May 2000)

\begin{abstract}
The phase diagram of the integer quantum Hall effect is numerically determined in the tight-binding model, which can account for overall features of recently obtained experimental phase diagram with direct transitions from high plateau states to the insulator. In particular, the quantum Hall plateaus are terminated by two distinct insulating regimes, characterized by the Hall resistance with classic and quantized values, respectively, which is also in good agreement with experiments. The physical origin of this peculiar phase diagram is discussed.
\end{abstract}

The previously proposed $^{1}$ theoretical global phase diagram (GPD) of the quantum Hall $(\mathrm{QH})$ effect predicts that only the $\nu=1 \mathrm{QH}$ liquid state is adjacent to the insulator, while higher $\mathrm{QH}$ plateau states do not neighbor with the insulator as schematically illustrated in Fig. 1(a). As a result, a direct transition from higher $\mathrm{QH}$ plateau $(\nu>1)$ states to the insulator is prohibited. However, a series of recent experimental measurements ${ }^{2-4}$ have indicated a phase diagram with qualitatively different topology. Most recently, it has been established experimentally by Hilke et $a l^{5}$ that direct transitions from $\nu=1,2, \ldots, 7$ to the insulating phase can all take place as clearly shown in Fig. 1(b). These experiments have challenged the basic theoretical understanding of $\mathrm{QH}$ systems $^{6}$ at weak magnetic field. ${ }^{7}$

These QH liquid-insulator transitions have also exhibited distinct properties in different regimes. For example, the Hall resistance $\rho_{x y}$ remains quantized at $h / e^{2}$ (Refs. 8 and 9) in the transition region between the $\nu=1 \mathrm{QH}$ state and the insulator at strong magnetic field or low Landau-level (LL) filling factor $n_{\nu}$, even though the longitudinal resistance $\rho_{x x}$ already increases almost by one order of magnitude above the value $h / e^{2}$ in the same region denoted as II in Fig. 1(b). By contrast, at weak magnetic field or higher $n_{\nu}, \rho_{x y}$ near the critical region of the $\nu>1 \mathrm{QH}$ states to insulator transition becomes $n_{\nu}$ dependent ${ }^{3}$ and is very close to the classic value $B /$ nec rather than the quantized value. In fact, such a classic behavior of $\rho_{x y}$ has been found ${ }^{2,8}$ to persist well into an insulating regime designated by I in Fig, 1(b), which suggests that there are two distinct insulating regimes surrounding the $\mathrm{QH}$ liquid states as opposed to the single one. ${ }^{1}$

Direct transitions between the $\nu>1 \mathrm{QH}$ states and the insulator have been already found in the tight-binding model (TBM) based on numerical calculations. ${ }^{10-12}$ But the relevance of such a lattice model to the experiment is still controversial, ${ }^{13}$ since the strength of the magnetic field usually cannot be reduced weak enough to directly simulate the realistic situation within the numerical capacity. Therefore, it is particularly important to identify the overall phase diagram and corresponding transport properties in such a model in order to understand the underlying physics and establish a real connection with the experiments.

In this paper, we obtain a numerical "global phase diagram' for the integer QH effect based on the TBM and the results are summarized in Fig. 1(c). The topology of the phase diagram is strikingly similar to the experimental one shown in Fig. 1(b), and in particular the insulating phase is indeed divided into two regimes: In a strong disorder and low-magnetic-field region (insulator I), the Hall resistance follows a classic value while the longitudinal resistance shows insulating behavior; in a weak disorder and highmagnetic-field region (insulator II), we find that $\rho_{x y}$ remains at the quantized value $h / e^{2}$ near the transition region even when $\rho_{x x}$ increases up to $8 h / e^{2}$. Both are in good agreement with the aforementioned experiments. Finally we provide a physical interpretation of the nature of the present nonfloat-up phase diagram based on the calculation of the equilibrium edge current.

The phase diagram in Fig. 1(c) can be determined by following the trace of extended levels by continuously tuning the disorder strength or magnetic field $B$. The position of each extended level forms a boundary that separates a given $\mathrm{QH}$ plateau state from another $\mathrm{QH}$ state or the insulating phase, and can be identified by the peak (which is sample size independent) of the density of states carrying a nonzero Chern number, ${ }^{10,13}$ calculated based on the TBM Hamiltonian $H=-\Sigma_{\langle i j\rangle} e^{i a_{i j}} c_{i}^{+} c_{j}+$ H.c. $+\sum_{i} w_{i} c_{i}^{+} c_{i}$, which is characterized by two parameters: the magnetic flux per plaquette $\phi=\Sigma_{\square} a_{i j}=2 \pi / M$ and the disorder strength $W$ of the random uncorrelated disorder potential $w_{i}:\left|w_{i}\right|<W / 2$. The result shown in Fig. 1(c) is calculated at $M=64$. In the Chern number calculation, the sample size is up to $64 \times 64$. The position of an extended level can be equally identified by the peak of the longitudinal conductance $\sigma_{x x}$, which coincides with the Chern number result, but this latter method has an advantage as it can be applied to much weaker magnetic fields. At $M=384$, the sample size in calculating $\sigma_{x x}$ is up to $L_{x}=200$ and $L_{y}=10^{3} M$ using the transfer-matrix method. ${ }^{14}$ The phase diagram in Fig. 1(c) remains essentially the same as we continuously change the magnetic flux from $M=8$ to 384 . Note that $W_{c}$ (which depends on the magnetic field $B$ ) is the critical value at which the last $\mathrm{QH}$ plateau state disappears and the system becomes an insulator.

The similarity between the numerical phase diagram [Fig. 1(c)] and the experimental one [Fig. 1(b)] is striking. A similar experimental phase diagram is also obtained earlier in Ref. 2. Several detailed features in Fig. 1(c) are worth mentioning. Firstly, starting from the strong-magnetic-field insulator II and reducing $B$ continuously at a fixed electron density, we obtain numerically a dashed curve $A$ shown in Fig. 1(c) that cuts through different phases with a transition pat- 

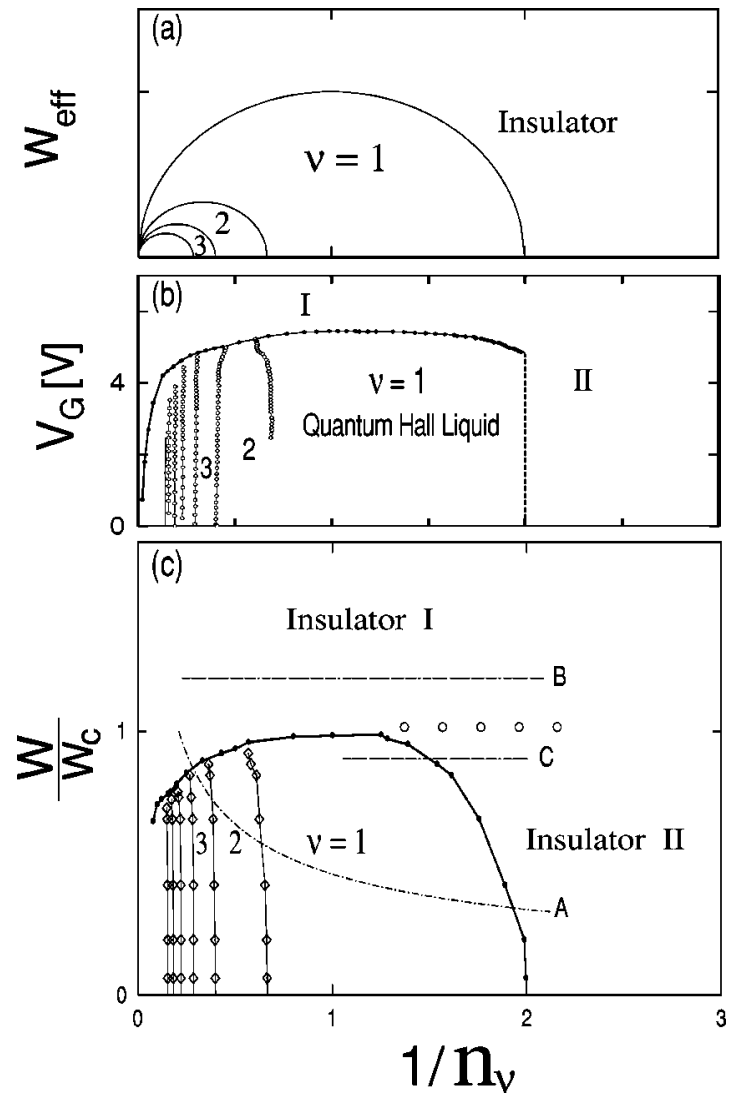

FIG. 1. The phase diagram in disorder vs $1 / n_{\nu}$ plane. (a) Theoretic global phase diagram predicted in Ref. 1. (b) Experimental one in Ref. 5. (c) The present numerical result. Note that the scan line $A$ in (c) corresponds to a constant $V_{G}$ line in (b) (see text).

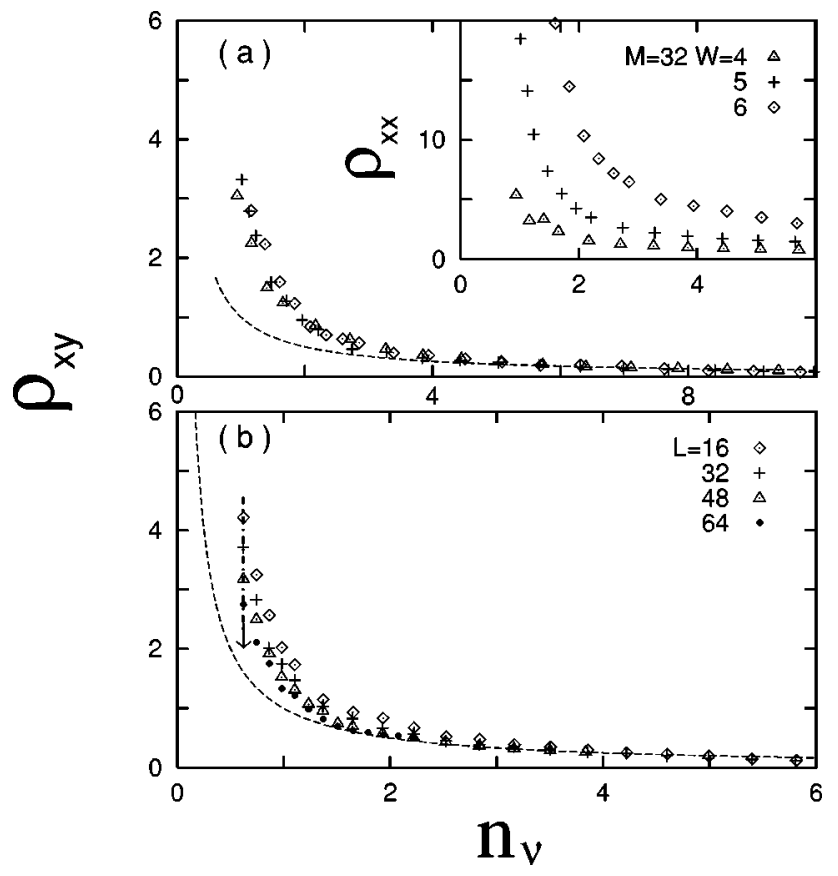

FIG. 2. Hall resistance $\rho_{x y}$ (in units of $h / e^{2}$ ) as a function of $n_{\nu}$ along the scan line B in Fig. 1(c). The dashed curve represents the classic value of $1 / n_{\nu}\left(h / e^{2}\right)=B / n e c$. (a) $\rho_{x y}$ at different disorder strength $W$ 's. The inset: $\rho_{x x}$ vs $n_{\nu}$. (b) The finite size effect of $\rho_{x y}$.

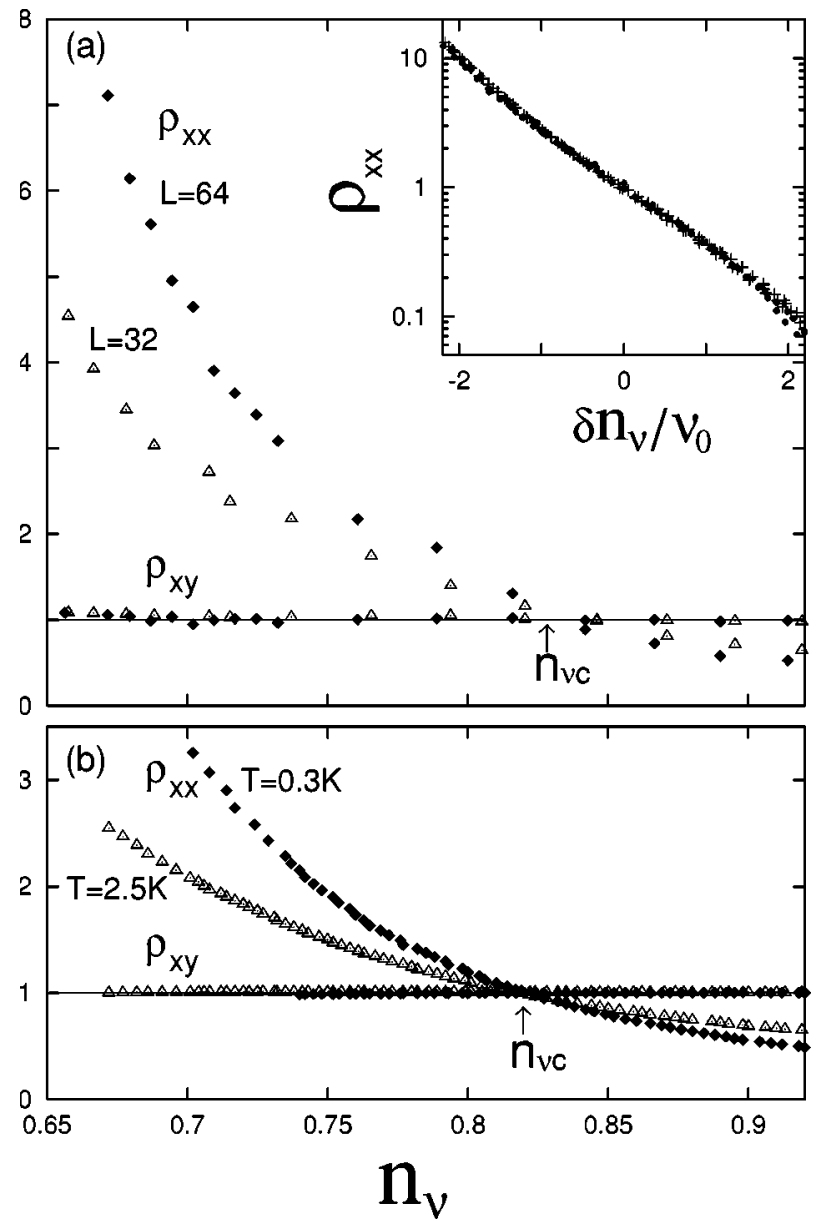

FIG. 3. (a) The longitudinal resistance $\rho_{x x}$ and Hall resistance $\rho_{x y}$ (in units of $h / e^{2}$ ) versus $n_{\nu}$ along the scan line C in Fig. 1(c) at $M=8$. The inset: The scaling function $\rho_{x x}=f\left(\delta n_{\nu} / \nu_{0}\right)$ obtained from the numerical calculation $(+)$ and the experimental measurement (Fig. 3 of Ref. 19) (O). (b) Experimental data (Ref. 8) at different temperatures (note that $\rho_{x x}$ is in units of $\rho_{c}=1.73 \mathrm{~h} / \mathrm{e}^{2}$ according to Ref. 8).

tern $0-1-2-3-0$. [As $B$ is reduced, $W / W_{c}(B)$ increases due to the $B$ dependence of $W_{c}$ ]. Such a scan curve should be equivalent to a constant gate voltage $V_{G}$ line in Fig. 1(b) as the fixed $V_{G}$ means both the disorder strength and electron density are constants. Secondly, one can clearly see that all the higher extended levels as boundaries separating different QH states are almost vertical lines in Fig. 1(c) which do not "float up" much in terms of the LL filling number $n_{\nu}$ at increasing disorder strength. The same non-float-up picture also unequivocally shows in the experimental phase diagram of Fig. 1(b). Only the lowest one which defines the boundary between $\nu=1$ and the insulator floats up, also in agreement with an earlier experiment. ${ }^{15}$

The scan curve $A$ in Fig. 1(c) connects insulating regimes at two ends. Let us first focus on the insulating region I that has a boundary neighboring with the high-plateau $\mathrm{QH}$ states. Figure 2 shows the calculated $\rho_{x x}$ and $\rho_{x y}$ versus $n_{\nu}$ at fixed $W$ [parallel to the scan line B illustrated in Fig. 1(c)]. The prominent feature in this region is that $\rho_{x y}$ follows the classic behavior [the dashed curve in Fig. 2 denotes $\left(1 / n_{\nu}\right)\left(h / e^{2}\right)$ $=B / n e c]$ : In Fig. 2(a), the magnetic field is fixed at $M$ $=32$ while different disorder strengths are considered. Even 


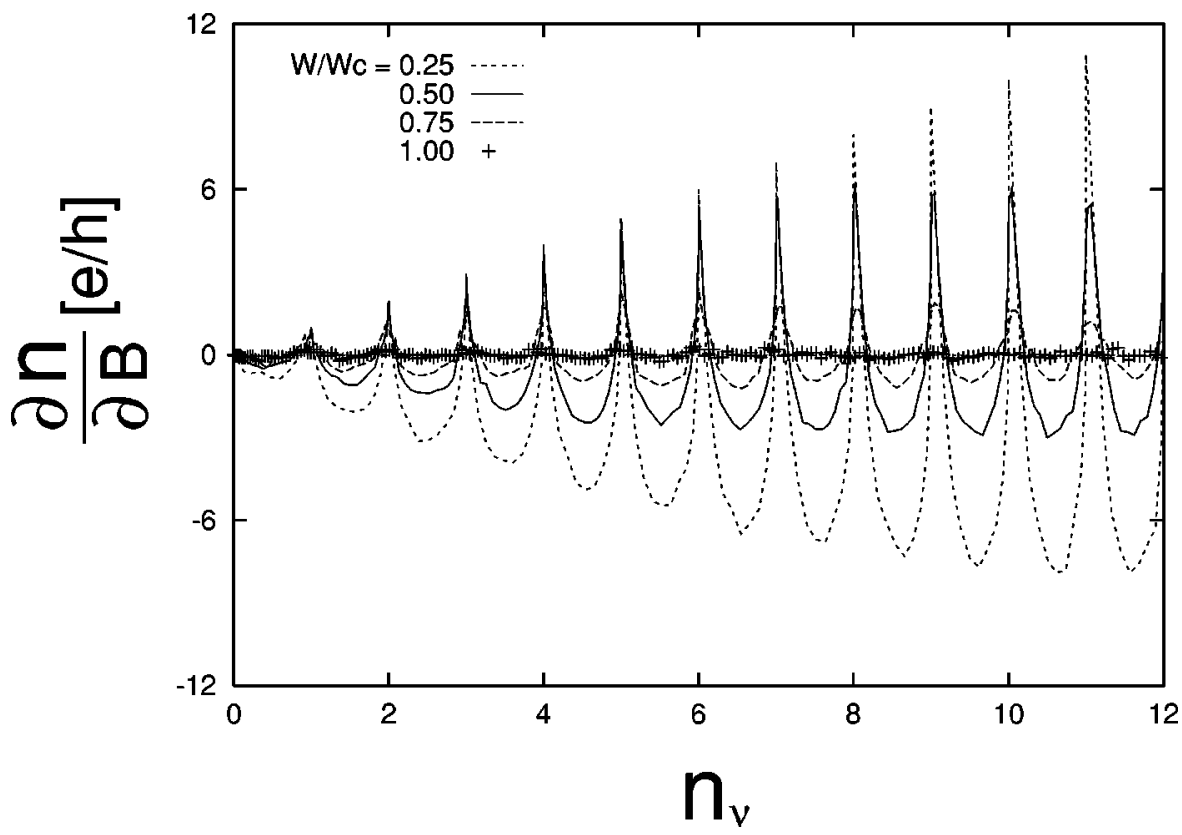

FIG. 4. The equilibrim edge current (Ref. 24) $\partial n /\left.\partial B\right|_{E_{f}}$ vs $n_{\nu}$. It indicates that the $\mathrm{QH}$ plateaus are pinned at integer $n_{\nu}{ }^{\prime} s$ until their destruction by disorder. though $\rho_{x x}$ in the inset grows with $W$ very quickly, $\rho_{x y}$ is insensitive to disorders and remains close to the classical value at $W=4,5,6 \quad\left(W_{c}=3.5\right)$. The finite-size effect of $\rho_{x y}$ is shown in Fig. 2(b) at $M=16$. By increasing the sample length $L$ from 16 to 64 , one sees that $\rho_{x y}$ converges to the dashed curve (the classic value) very quickly whereas $\rho_{x x}$ keeps increasing monotonically with the sample size. It is noted that both $\sigma_{x x}$ and $\sigma_{x y}$ calculated here are for square samples $L \times L$ using Landauer ${ }^{14}$ and Kubo formulas, respectively, and more than 2000 disorder configurations are taken at $L=64$ and even more at smaller sample sizes.

Such a classic behavior of $\rho_{x y}$ has been extensively observed experimentally ${ }^{2,3,8}$ in the weak-magnetic-field regime. $\rho_{x y} \propto 1 / n_{\nu}$ in fact still holds at the critical point between the $\mathrm{QH}$ liquid and insulating regime I as previously shown experimentally $^{3}$ and numerically. ${ }^{16}$ Since LL's are effectively coupled together at weak field and strong disorders, we believe that this phenomenon reflects the fact that the regime I is basically an Anderson insulator: $\rho_{x y}$ is always unrenormalized and remains at the classic value. ${ }^{17}$ In other words, insulator I in Fig. 1(c) should continuously evolve into the well-known Anderson insulator at zero magnetic field without changing the classic behavior of $\rho_{x y}$ while $\rho_{x x}$ is always divergent in the thermodynamic limit at zero temperature.

Now we consider the insulating regime II in Fig. 1(c). Along the scan line $\mathrm{C}$ in Fig. $1(\mathrm{c})$, the results of $\rho_{x x}$ and $\rho_{x y}$ are presented in Fig. 3(a). It shows that $\rho_{x y}$ remains at quantized value $h / e^{2}$ while $\rho_{x x}$ arises almost an order of magnitude from the critical value at $n_{\nu c}$ into the insulator region. This is in contrast to the aforementioned classic behavior $\rho_{x y}=\left(1 / n_{\nu}\right) h / e^{2}$ in regime I. Such a quantized $\rho_{x y}$ exists in the whole critical region along the boundary between the $\nu$ $=1 \mathrm{QH}$ state and the insulator. The open circles in Fig. 1(c) at $W / W_{c}=1$ lies very close to the boundary of the two insulating regimes as indicated in our numerical calculations (how two regimes exactly cross over will need a more careful study which is beyond the scope of the present paper). It is noted that in the transition region where $\rho_{x y}=h / e^{2}$ is ob- served, both $\sigma_{x x}$ and $\sigma_{x y}$ satisfy a one parameter scaling law, ${ }^{18}$ which suggests that it is a consequence related to the quantum phase transition.

The experimental results ${ }^{8}$ of $\rho_{x x}$ and $\rho_{x y}$ are presented for comparison in Fig. 3(b). It shows that the range of $n_{\nu}$ for the quantized $\rho_{x y}$ and the corresponding values of $\rho_{x x}$ are very close to our numerical ones. Here the temperature dependence of the experimental data can be translated into the $L$ dependence of our numerical results at $T=0$ through a dephasing length $L_{i n}$. To further compare with the experiments, ${ }^{19,20}$ the calculated $\rho_{x x}$ as a scaling function of the relative LL filling number $\delta n_{\nu}=n_{\nu}-n_{\nu c}$, i.e., $\rho_{x x}$ $=f\left(\delta n_{L} / \nu_{0}\right)$, is shown in the inset of Fig. 3(a), where $\nu_{0}$ $=c_{0}\left(L / l_{0}\right)^{-1 / x}, x=2.3$, and $l_{0}$ is the magnetic length $\left(c_{0}\right.$ is a dimensionless constant). The experimental data (from Fig. 3 of Ref. 19) are also plotted in the inset using the $T$-dependent $\nu_{0}$ and an excellent agreement over a wide range of the scaling variables: $-2<\delta n_{\nu} / \nu_{0}<2$ is clearly shown.

Here we note that in some experiments ${ }^{9}$ whether the quantum critical regime is reached is still controversial and there is an alternative explanation for the quantized $\rho_{x y}$ regime in which interactions may play $^{21}$ a crucial role for a nonscaling behavior of the transport coefficients. An important distinction between such an interaction case and the present theory is that in the former case $\rho_{x y}$ is always well quantized in the insulating regime while the quantization of $\rho_{x y}$ in Fig. 3(a) is mainly confined around the critical point $n_{\nu c}$ with $\rho_{x x}<10 h / e^{2}$ and $\rho_{x y}$ eventually will start to distinctly grow with $\rho_{x x}$ as $\rho_{x x}$ further increases. ${ }^{22}$ Further experimental measurement in this regime may help to clarify this issue.

Finally, we would like to discuss a key physical distinction between the numerical phase diagram in Fig. 1(c) and the GPD in Fig. 1(a). In the latter case, all the QH boundaries eventually float up to $n_{\nu} \rightarrow \infty$ at $B \rightarrow 0$ with the LL plateau structure in between remaining basically unchanged. But in both the TBM and the experiments, those vertical $\nu>1 \mathrm{QH}$ boundaries [see Fig. 1(c)] do not markedly "float up" in $n_{\nu}$ with increasing $W$ or reducing $B$ such that each LL plateau in 
between never floats away: only the width of the $\nu$ th $\mathrm{QH}$ plateau is reduced and vanishes eventually at the $\nu \rightarrow 0$ transition boundary. It then results in direct transitions and two insulating regimes in Fig. 1(c). A recent numerical work ${ }^{23}$ using short-range correlated disorders to smooth the lattice effect has revealed that the non-float-up nature of plateaus is robust and can survive at extremely weak magnetic fields. To further understand this picture, we calculate the so-called equilibrium edge current ${ }^{24}$ which is proportional to $\partial n /\left.\partial B\right|_{E_{f}}$ (Ref. 25) ( $n$ is the electron density and $E_{f}$ is the Fermi energy) and is $L$ independent. The results (which are $B$-independent) are present in Fig. 4 in which the peaks determine the centers of QH plateaus. ${ }^{24}$ Indeed, such a quantity is continuously reduced with increasing $W$ and eventually diminishes at $W_{c}$, but its peak positions at $W<W_{c}$ never move away which clearly indicates that the recovery of an Anderson insulator at strong disorder in the integer QH system is due to the destruction of the plateaus instead of a float up of the whole QH structure towards $n_{\nu} \rightarrow \infty$.
To summarize, we have determined a numerical phase diagram of the integer $\mathrm{QH}$ based on the TBM. The topology of such a phase diagram is remarkably similar to the experimental one obtained recently for the $\mathrm{QH}$ system. Two kinds of insulating regimes surrounding the $\mathrm{QH}$ plateau phase are identified whose transport properties, characterized by the classic and quantized values of the Hall resistance, respectively, are also in good agreement with the experiments. The nature of such a phase diagram can be understood as a continuous narrowing and collapsing of the $\mathrm{QH}$ plateaus which are pinned around discrete LL filling numbers without floating away.

The authors would like to acknowledge helpful discussions with R. N. Bhatt, S. V. Kravchenko, X.-G. Wen, L. P. Pryadko, A. Auerbach, and especially P. Coleridge and M. Hilke who also provided us their experimental data prior to publication. This work was supported by the State of Texas through ARP Grant No. 3652707 and the Texas Center for Superconductivity at University of Houston.
${ }^{1}$ S. Kivelson, D. H. Lee, and S. C. Zhang, Phys. Rev. B 46, 2223 (1992).

${ }^{2}$ S. V. Kravchenko, W. Mason, J. E. Furneaux, and V. M. Pudalov, Phys. Rev. Lett. 75, 910 (1995); A. A. Shashkin, G. V. Kravchenko, and V. T. Dolgopolov, [Pis'ma Zh. Éksp. Teor. Fiz. 58, 215 (1993) [JETP Lett. 58, 220 (1993)]; V. M. Pudalov et al., Surf. Sci. 305, 107 (1994); Phys. Lett. B 194, 1287 (1994).

${ }^{3}$ S. -H. Song, D. Shahar, D. C. Tsui, Y. H. Xie, and D. Monroe, Phys. Rev. Lett. 78, 2200 (1997); D. Shahar, D. C. Tsui, and J. E. Cunningham, Phys. Rev. B 52, R14 372 (1995).

${ }^{4}$ C. H. Lee, Y. H. Chang, Y. W. Suen, and H. H. Lin, Phys. Rev. B 58, 10629 (1998).

${ }^{5}$ M. Hilke, D. Shahar, S. H. Song, D. C. Tsui, and Y. H. Xie, Phys. Rev. B (to be published).

${ }^{6}$ For reviews see, The Quantum Hall Effect, edited by R. E. Prange and S. M. Girvin (Springer-Verlag, New York, 1990).

${ }^{7}$ R. B. Laughlin, Phys. Rev. Lett. 52, 2304 (1984); D. E. Khmel'nitzkii, Phys. Lett. 106A, 182 (1984).

${ }^{8}$ M. Hilke, D. Shahar, S. H. Song, D. C. Tsui, and Y. H. Xie, and D. Monroe, Nature (London) 395, 675 (1998).

${ }^{9}$ D. Shahar, M. Hilke, C. C. Li, D. C. Tsui, S. L. Sondhi, J. E. Cunningham, and M. Razeghi, Solid State Commun. 107, 19 (1998); D. Shahar, D. C. Tsui, M. Shayegan, E. Shimshoni, and S. L. Songhi, Phys. Rev. Lett. 79, 479 (1997); Science 274, 589 (1996); M. Hilke et al., Europhys. Lett. (to be published); D. Shahar, S. H. Song, D. C. Tsui, Y. H. Xie, and Don Monroe, Phys. Rev. B 56, 15545 (1997).

${ }^{10}$ D. N. Sheng and Z. Y. Weng, Phys. Rev. Lett. 78, 318 (1997).
${ }^{11}$ D. Z. Liu, X. C. Xie, and Q. Niu, Phys. Rev. Lett. 76, 975 (1996); X. C. Xie, D. Z. Liu, B. Sundaram, and Q. Niu, Phys. Rev. B 54, 4966 (1996).

${ }^{12}$ H. Potempa, A. Baker, and L. Schweitzer, Physica B 256, 591 (1998); Y. Hatsugai, K. Ishibashi, and Y. Morita, Phys. Rev. Lett. 83, 2246 (1999).

${ }^{13}$ K. Yang and R.N. Bhatt, Phys. Rev. B 59, 8144 (1999); Phys. Rev. Lett. 76, 1316 (1996).

${ }^{14}$ A. MacKinnon and B. Kramer, Z. Phys. 53, 1 (1983); D. S. Fisher and P. A. Lee, Phys. Rev. B 23, 6851 (1981).

${ }^{15}$ I. Glozman, C. E. Johnson, and H. W. Jiang, Phys. Rev. Lett. 74, 594 (1995).

${ }^{16}$ D. N. Sheng and Z. Y. Weng, Phys. Rev. Lett. 80, 580 (1998).

${ }^{17}$ P. A. Lee and T. V. Ramakrishnan, Rev. Mod. Phys. 57, 287 (1985).

${ }^{18}$ B. Huckestein and B. Kramer, Phys. Rev. Lett. 64, 1437 (1990); B. Huckestein, Rev. Mod. Phys. 67, 357 (1995).

${ }^{19}$ P. T. Coleridge and P. Zawadzki, cond-mat/9903246 (unpublished).

${ }^{20}$ R. T. F. van Schaijk, A. de Visser, S. M. Olsthoorn, H. P. Wei, and A. M. M. Pruisken, Phys. Rev. Lett. 84, 1567 (2000).

${ }^{21}$ E. Shimshoni and A. Auerbach, Phys. Rev. B 55, 9817 (1997); L. P. Pryadko and A. Auerbach, Phys. Rev. Lett. 82, 1253 (1999).

${ }^{22}$ D. N. Sheng and Z. Y. Weng, Phys. Rev. B 59, R7821 (1999).

${ }^{23}$ D. N. Sheng, Z. Y. Weng, and X. G. Wen, cond-mat/0003117 (unpublished).

${ }^{24}$ A. M. M. Pruisken, in The Quantum Hall Effect, Ref. 6.

${ }^{25} \partial n /\left.\partial B\right|_{E_{f}}=\int E_{f}\left[\rho_{b}(B+\Delta B)-\rho_{b}(B)\right] d E / \Delta B(\Delta B \ll B)$ with $\rho_{b}$ as the bulk density of states, see also Ref. 24 . 